\title{
The value of interleukin- 6 (IL-6) within 6 hours after birth in the prompt diagnosis of early-onset neonatal sepsis
}

\author{
Chunmei Liu^, Chengzhi Fang, Qi He, Lili Xie \\ Department of Neonatology, Renmin Hospital of Wuhan University \& Hubei General Hospital, Wuhan, China \\ Contributions: (I) Conception and design: C Liu; (II) Administrative support: C Liu; (III) Provision of study materials or patients: All authors; (IV) \\ Collection and assembly of data: All authors; (V) Data analysis and interpretation: All authors; (VI) Manuscript writing: All authors; (VII) Final \\ approval of manuscript: All authors. \\ Correspondence to: Chunmei Liu. Department of Neonatology, Renmin Hospital of Wuhan University and Hubei General Hospital, Wuhan, China. \\ Email: liuchunmei123@126.com.
}

Background: To investigate the value of interleukin-6 (IL-6) for neonates within 6 hours after birth in the prompt diagnosis of early-onset neonatal sepsis (EONS).

Methods: The clinical and laboratory data of 129 neonates in the neonatal intensive care unit (NICU) of our center from March 31, 2017, to February 29, 2020, were retrospectively analyzed. These patients were divided into two groups on their disease conditions: the EONS group $(n=66)$ and the healthy control group ( $\mathrm{n}=63$ ). All enrolled patients were born in our hospital's Obstetrics Department and were admitted to the NICU within 2 hours after birth. The first session of the blood test was conducted within 4-6 hours after birth for the measurements of IL-6, C-reactive protein (CRP1), serum amyloid A1 (SAA1), and serum immunoglobulin M (IgM). The second session of the blood test was performed 12-24 hours after birth for procalcitonin (PCT), CRP2, and SAA2. All the tests were completed in our clinical laboratory. The nonparametric test (Mann-Whitney $U$ test) was used to compare all parameters between these two groups. The receiver operating characteristic (ROC) curves were drawn to compare the diagnostic sensitivities and specificities. The pairwise comparisons of the ROC curves were on the MedCalc18.2.1 software. A P value of $<0.05$ was considered statistically significant.

Results: Gender, birth weight, and gestational age were matched between the EONS group and the control group (all P>0.05). The differences of IL-6, CRP2, PCT, and SAA2 were statistically significant between these two groups (all $\mathrm{P}<0.05$ ), while there was no significant difference in CRP1, SAA1, and IgM (all P>0.05). The area under the ROC curve (AUC) is 1 (95\% CI: 0.918-1.000), 1 (95\% CI: 0.918-1.000), 1 (95\% CI: 0.918-1.000), and 0.977 (95\% CI: 0.878-0.999), respectively, for IL-6, CRP2, PCT, and SAA2. Pairwise comparisons among four biomarkers showed the diagnostic specificity and sensitivity of IL-6 were not significantly different from those of CRP2, PCT, and SAA2 (all P>0.05).

Conclusions: IL-6 is a quick and independent diagnostic biomarker for EONS, and its sensitivity and specificity are inferior to the conventional inflammation markers, including CRP, PCT, and SAA.

Keywords: Interleukin-6 (IL-6); early-onset neonatal sepsis (EONS); early diagnosis

Submitted Jul 03, 2020. Accepted for publication Sep 30, 2020.

doi: $10.21037 /$ tp-20-239

View this article at: http://dx.doi.org/10.21037/tp-20-239

$\wedge$ ORCID: 0000-0001-2345-6789. 


\section{Introduction}

Neonatal sepsis is a systemic infection occurring in neonates $\leq 28$ days old. According to the age of onset, it is divided into early-onset neonatal sepsis (EONS; aged $\leq 72 \mathrm{~h}$ ) and late-onset neonatal sepsis (LONS; aged $>72 \mathrm{~h}$ ) (1). Despite its non-specific clinical symptoms, EONS is highly fatal and can lead to serious long-term complications. Unfortunately, the gold-standard blood culture technique requires at least 24-48 hours, and the prenatal use of antibiotics further decreases the accuracy of blood culture.

Inflammatory factors, including C-reactive protein (CRP), serum amyloid A protein (SAA), and procalcitonin (PCT), are often used to assist in the diagnosis of EONS. CRP is one of the most widely studied and applied acute phase proteins clinically, which can be induced by pre-inflammatory factor interleukin-6 (IL-6) to synthesize by liver cells, and it starts to rise in 12-24 hours of inflammatory response and reaches its peak at 48 hours. PCT is a non-hormone active precalcitonin peptide, which can be induced by IL-6 during the inflammatory reaction. It starts to rise in 3-4 hours and reaches its peak in $18-24$ hours. SAA is a precursor of serum amyloid A, and IL-6 can also mainly induce liver production earlier than CRP, which can be significantly increased 48 hours later, proved to be superior to CRP in the diagnosis of EONS (2). Again, although these biomarkers have an absolute specificity and sensitivity for the diagnosis of EONS, they cannot make a definite diagnosis promptly. Thus, a biomarker that does not depend on blood culture is urgently needed to enable prompt diagnosis and guide the treatment. IL- 6 is a pro-inflammatory cytokine secreted by mononuclear macrophages after a specific pathogen activates the toll-like receptors (TLRs) in the early stages of the inflammatory response (3). IL-6 is a preinflammatory cytokine that has been extensively studied in EONS. It is stimulated by B and T lymphocytes at the onset of inflammatory responses. Its level rises 2 hours after infection and peaks at 4-6 hours (4). This induces hepatocytes to produce PCT and acute reactants including CRP and SAA $(5,6)$. Therefore, it is speculated that IL-6 is much more significant than CRP and PCT and SAA in the early detection of infection. It has also been found that the abnormal IL-6 elevation in maternal serum can be used as an independent factor to predict intra-amniotic infection (7). EONS are closely related to chorioamnionitis; studies have confirmed that IL-6 plays a crucial role in the pathogenesis of fetal and neonatal inflammatory diseases, including sepsis, bronchopulmonary dysplasia, and necrotizing enterocolitis
$(8,9)$. Thus, it is hypothesized, which IL-6 may also be an independent diagnostic predictor of EONS.

Here, we retrospectively analyzed the IL-6 level in EONS patients within 6 hours after birth and compared it with several commonly-used inflammatory factors, with an attempt to identify the independent diagnostic value of IL-6 in EONS and thus facilitate the early treatment of this disease. We attempted to establish the independent diagnostic value of IL-6 as an independent biological indicator for EONS, i.e., definite diagnosis and antimicrobial therapy within 6 hours after birth. However, little research has been done on IL-6 in this regard. It has been well recognized that early diagnosis and early treatment are critical strategies for lowering the mortality and disability rates of EONS $(10,11)$, and few studies have investigated the role of IL-6 in the initial stage after birth.

We present the following article in accordance with the STARD reporting checklist (available at http://dx. doi. org/10. 21037/tp-20-239).

\section{Methods}

The study followed the Helsinki Declaration (as revised in 2013) and was approved by the Ethics Committee of People's Hospital of Wuhan University (The Ethics Committee of our school agreed, but no number was given because there was no relevant project funding for this article). All the children in our department were treated with the informed consent of their guardians.

\section{Subjects}

A total of 129 neonates cared in the neonatal intensive care unit (NICU) of our center from March 31, 2017, to February 29, 2020, were selected for this retrospective study. All the cases were admitted to NICU within 2 hours after birth in the obstetrics department. The gestational age of these neonates ranged 28-34 weeks, and the gender, body weight, and gestational age showed no significant difference between the EONS group $(n=66)$ and the control group ( $\mathrm{n}=63)$ (Table 1). Cases in the EONS group met the following diagnostic criteria for sepsis (11): (I) the suspected cases had any of the following features within three days of being born: (i) with abnormal clinical manifestations; (ii) the mother had chorioamnionitis; (iii) with premature rupture of membranes (PROM) $\geq 18$ hours before the onset of the contractions. If there was no abnormal clinical manifestation, blood culture was 
Table 1 General data of subjects in two groups

\begin{tabular}{lccc}
\hline Variables & $\begin{array}{c}\text { EONS group } \\
(\mathrm{n}=66)\end{array}$ & $\begin{array}{c}\text { Control group } \\
(\mathrm{n}=63)\end{array}$ & P value \\
\hline Birth weight $(\mathrm{g})$ & $1,753 \pm 346$ & $1,746 \pm 401$ & $>0.05$ \\
Gestational age $(\mathrm{w})$ & $31.5 \pm 3.2$ & $31.2 \pm 3.1$ & $>0.05$ \\
Gender $(\mathrm{s}, \mathrm{M} / \mathrm{F})$ & $32 / 34$ & $31 / 32$ & $>0.05$ \\
\hline
\end{tabular}

negative, and two consecutive blood non-specific tests (with a 24-hour interval) showed less than two positive results, the possibility of sepsis was ruled out. (II) A clinical diagnosis was made if a patient had abnormal clinical manifestations and meanwhile met any of the following criteria: (i) blood non-specific test showed two or more positive results; (ii) cerebrospinal fluid test revealed purulent meningitis; (iii) bacterial DNA in blood. (III) A diagnosis of EONS was confirmed if there were typical clinical manifestations of sepsis and positive findings in blood/cerebrospinal fluid (or other sterile cavity fluid) cultures. In the EONS group, EONS was the primary diagnosis, while in the control group, premature infant was the primary diagnosis. The enrolled patients who had obvious deformity, with Apgar score $<7$ and those who had used antibiotics before the start of the study were excluded from this study.

Typically, IL-6 rises 2 hours after infection and reaches a peak at 4-6 hours; PCT, CRP, and SAA began to rise 6 hours after birth and reach their peaks at 12-24 hours (3). $\operatorname{IgM}$ has been used as one of the predictive indicators of intrauterine infection (12). Thus, we checked these biomarkers early after birth and compared them with IL-6. For the secretion peaks of these biomarkers, we collected blood samples within 4-6 hours after birth (before using antibiotics) for blood culture and the measurements of IL-6, CRP1, and SAA1; 12-24 hours after birth, we collected the second blood samples for both routine blood test and PCT/CRP2/SAA2 measurements. All the tests were completed within 24 hours after birth.

\section{Measurements}

Blood culture was completed in an automatic microbiological detection system (BacT/ALERT3D60, Bio Mérieux) and a 24-hour early warning system. A preliminary culture report was generated 3 days (72 hours) after culture for bacteria, and a final culture report was obtained on day 5 (120 hours). IL-6,
CRP, SAA, and IgM were determined by the enzymelinked immunosorbent assay (ELISA: Booster Biological Technology Co. Ltd. Wuhan, China), and PCT was measured using electrochemical luminescence immunoassay (E601, Roche Cobas). The measurement results of IL-6, PCT, CRP, and SAA were obtained within 1 hour. All the tests were completed in our clinical laboratory.

\section{Data analysis}

The non-parametric test (Mann-Whitney U test) was used to compare all parameters between groups. The receiver operating characteristic (ROC) curves were drawn to compare the diagnostic sensitivities and specificities, and the areas under the ROC curve (AUC) were calculated. The pairwise comparisons of the ROC curves were on the MedCalc18.2.1 software. A $P$ value of $<0.05$ was considered statistically significant.

\section{Results}

\section{General data}

There were no significant differences in the gender, weight, and gestational age between the EONS group and the control group $(\mathrm{P}>0.05)$. All the cases in the EONS group met the diagnostic criteria for EONS (13) (Table 1).

The measurement results of IL-6, PCT, CRP1, SAA1, CRP2, SAA2, and IgM, are listed in Table 2.

Results of AUROC analysis for IL-6, PCT, CRP1, CRP2, SAA1, and SAA2 between the two groups are shown in Figure 1 and Table 3.

As shown in Table 1, there were no significant differences in the gender, weight, and gestational age between the EONS group and the control group $(\mathrm{P}>0.05)$. Since the data were not normally distributed in both groups, a nonparametric test (Mann-Whitney $U$ test) was performed in the SPSS20.0 software package. It was found the distribution of CRP1, SAA1, and IgM in the EONS group and the control group was not statistically different (all $\mathrm{P}>0.05$ ), whereas the distribution of CRP2, IL-6, PCT, and SAA2 showed significant difference (all $\mathrm{P}<0.05$ ) (Table 2). Pairwise comparisons of the sensitivities and specificities of IL-6, CRP2, SAA2, and PCT between the EONS group and the control group in the MedCalc software (version 18.2.1) on ROC curves (Figure 1) showed no significant difference $(\mathrm{P}>0.05)$ (Table 3$)$. 
Table 2 Comparisons of the mean rank orders of IL-6, CRP1, CRP2, SAA1, SAA2, PCT, and IgM between the two groups

\begin{tabular}{lcccc}
\hline Group & G1 & G2 & $U$ & $\mathrm{P}$ \\
\hline $\mathrm{n}$ & 66 & 63 & - & - \\
$\mathrm{IL}-6$ & 32.5 & 11 & 0 & $<0.01$ \\
CRP1 & 22.95 & 21 & 210 & 0.311 \\
CRP2 & 32.5 & 11 & 0 & $<0.01$ \\
SAA1 & 22.95 & 21 & 210 & 0.162 \\
SAA2 & 32.02 & 11.5 & 10.5 & $<0.01$ \\
PCT & 32.5 & 11 & 0 & $<0.01$ \\
IgM & 19.5 & 24.62 & 176 & 0.181 \\
\hline
\end{tabular}

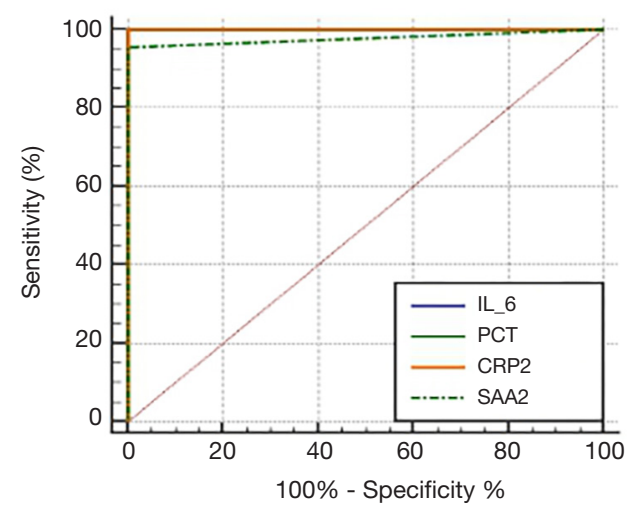

Figure 1 ROC curves for the diagnostic values of IL-6, PCT, CRP2, and SAA2. ROC, receiver operating characteristic.

\section{Discussion}

Our retrospective analysis showed that IL- 6 had independent diagnostic significance for EONS in the early postnatal period (within 6 hours after birth). To our knowledge, it was the first study on the value of IL-6 in diagnosing EONS in the initial stage after birth. EONS are closely related to intrauterine infection. Both the prenatal use of antibiotics and the low positivity rate during the time-consuming blood culture make it difficult to diagnose EONS early. EONS is a highly dangerous condition and can be resistant to antibiotics, and the final mortality and disability rates are high (14). Thus, more objective biomarkers are needed for early diagnosis and prompt treatment. The most commonly used biomarkers in diagnosing infection include CRP, PCT, and SAA
(15-17). Among them, PCT can physiologically rise after birth (18), and its diagnostic value for LONS is better than for EONS (19).

However, SAA increases more markedly in viral infections, and it is used as an indicator for distinguishing bacterial and viral infections (16). IL-6 is a proinflammatory factor, and the secretion of PCT, CRP, and SAA is later than IL-6 expression. It can be detected within 2 hours of infection and peaks at 4-6 hours (3). Studies in pregnant mothers and cord blood have demonstrated that IL-6 can be used as an independent factor for predicting intrauterine chorionitis (7), the most significant independent risk factor for EONS. It is, therefore, hypothesized that IL-6 might be closely related to EONS. In our current study, we determined the IL-6 level in neonates to investigate whether it could be used as an independent biomarker to diagnose EONS.

In this retrospective study, we found IL-6 increased significantly in the EONS group compared with the control group, consistent with a previous study (20). Considering the characteristics of various inflammatory factors in the inflammatory reaction (3) and the difficulty in blood collection in newborns, we measured IL-6 and IgM within 4-6 hours after birth and measured PCT within 12-24 hours after birth. Meanwhile, CRP and SAA were also determined. We found IL-6 was significantly increased in the EONS group in the initial stage (within 6 hours) after birth, while the CRP and SAA levels were not statistically different from those in the control group in the same period. We speculate that independent of CRP and SAA, IL-6 can reflect the body's inflammatory response at an extremely early stage after birth and has the possible value of diagnosing sepsis independently. Furthermore, PCT, CRP, and SAA in the EONS group showed significant differences with those in the control group. Also, in our current study, we found when IL-6 reached its peak during the inflammation, the PCT was still at its physiological peak, whereas CRP and SAA were not statistically different between the two groups. Since both PCT and IL-6 are the most-recommended biomarkers for diagnosing sepsis (21), it is inferred IL-6, which has the highest diagnostic value within 6 hours after birth. We also identified the optimal threshold of IL-6 in diagnosing EONS is $86.16 \mathrm{pg} / \mathrm{dL}$, which can be identified at the extremely early stage after birth. Comparisons of the AUROC curve of IL-6 with those of PCT, CRP, and SAA showed the diagnostic sensitivity 
Table 3 The diagnostic sensitivities and specificities of IL-6, CRP2, SAA2, and PCT in two groups

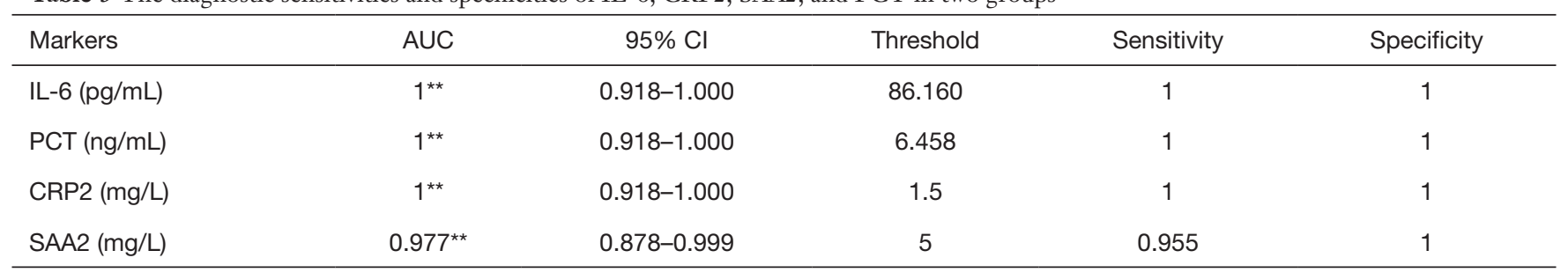

**, $\mathrm{P}<0.01$.

and specificity of IL-6 within 6 hours after birth showed no significant differences with those of routine markers including PCT, CRP, and SAA 12-24 hours after birth ( $\mathrm{P}>0.05)$. Thus, IL-6 may help make a preliminary diagnosis of EONS within 6 hours after birth, which is necessary for the clinical setting because literature has demonstrated each hour delay in antibiotic administration was associated with a $7.6 \%$ increase in mortality $(10,11)$.

Furthermore, we found the level of $\operatorname{IgM}$ was not significantly different between the EONS group and the control group within 6 hours after birth $(\mathrm{P}>0.05)$, while IL-6 showed significant differences at the same interval. It has been proposed that increased IgM suggests an intrauterine viral infection (22). However, we have found that EONS is closely related to intrauterine bacterial infections (1). Therefore, we hypothesize that IL-6 is better than $\operatorname{IgM}$ as a marker of intrauterine bacterial infection, and it is more feasible for the diagnosis of EONS.

On the features of inflammation markers during sepsis, Il-6 rises rapidly within 2 to 4 hours from the onset of inflammation, declines in the following 24 hours and drops drastically to undetectable levels after initiation of antibiotic therapy (4). With its Short half-life and the concentration declines drastically after antibiotic therapy, the detection window is very narrow. Although IL-6 is useful in monitoring host responses to infection and drug treatment, all the cases of the EONS group were treated with antibiotics Within 6 hours after birth, so according to its characteristics makes little sense to monitor continuously. Therefore, continuous IL-6 monitoring is not routinely performed clinically. According to the characteristics of the response during the inflammatory process, we measured IL-6, PCT, CRP, and SAA levels at different time intervals to avoid human errors. We attempt to monitorIL-6 continuously and CRP, SAA and PCT before and after antibiotic therapy of the EONS group clinically, because of IL-6 short half- life period and declining significantly after antibiotic treatment, which leads to limitations of clinical monitoring with a short inspection window period. However, CRP, SAA and PCT have a relatively long half-life, predict they can serve as good monitoring indicators, for guiding antibiotic treatment for further research. However, because of the small sample size, as our hospital is a center for critically ill pregnant women in Wuhan and even the Mid-South Region, the patients come from all over the province and surrounding provinces. The abundant case resources which solve the limitation of a small sample size to some extent, further analysis and confirmation of large samples and multi-center data are needed.

In conclusion, our study found the IL-6 level remarkably differed between EONS neonates and health controls. Its threshold can be easily identified in an extremely early stage after birth; meanwhile, its diagnostic sensitivity and specificity were inferior to those of CRP, PCT, and SAA after 12-24 hours. Therefore, IL-6 can be used as an independent biomarker for diagnosing EONS at the initial stage after birth.

\section{Acknowledgments}

Funding: None.

\section{Footnote}

Reporting Checklist: The authors have completed the STARD reporting checklist. Available at http://dx. doi. org/10. 21037/tp-20-239

Data Sharing Statement: Available at http://dx. doi. org/10.21037/tp-20-239

Conflicts of Interest: All authors have completed the ICMJE 
uniform disclosure form (available at http://dx. doi. org/10.21037/tp-20-239). The authors have no conflicts of interest to declare.

Ethical Statement: The authors are accountable for all aspects of the work in ensuring that questions related to the accuracy or integrity of any part of the work are appropriately investigated and resolved. The study followed the Helsinki Declaration (as revised in 2013) and was approved by the Ethics Committee of People's Hospital of Wuhan University (The Ethics Committee of our school agreed, but no number was given because there was no relevant project funding for this article). All the children in our department were treated with the informed consent of their guardians.

Open Access Statement: This is an Open Access article distributed in accordance with the Creative Commons Attribution-NonCommercial-NoDerivs 4.0 International License (CC BY-NC-ND 4.0), which permits the noncommercial replication and distribution of the article with the strict proviso that no changes or edits are made and the original work is properly cited (including links to both the formal publication through the relevant DOI and the license). See: https://creativecommons.org/licenses/by-nc-nd/4.0/.

\section{References}

1. Adams M, Bassler D. Practice variations and rates of late onset sepsis and necrotizing enterocolitis in very preterm born infants, a review. Transl Pediatr 2019;8:212-26.

2. Yuan H, Huang J, Lv B, et al. Diagnosis value of the serum amyloid A test in neonatal sepsis: a meta-analysis. Biomed Res Int 2013;2013:520294.

3. Janeway CA Jr, Medzhitov R. Innate immune recognition. Annu Rev Immunol 2002;20:197-216.

4. Chiesa C, Pacifico L, Natale F, et al. Fetal and early neonatal interleukin-6 response. Cytokine 2015;76:1-12.

5. Pavcnik-Arnol M, Hojker S, Derganc $M$. Lipopolysaccharide-binding protein in critically ill neonates and children with suspected infection: comparison with procalcitonin, interleukin-6, and C-reactive protein. Intensive Care Med 2004;30:1454-60.

6. Kishimoto T. The biology of interleukin-6. Blood 1989;74:1-10.

7. Park H, Park KH, Kim YM, et al. Plasma inflammatory and immune proteins as predictors of intra-amniotic infection and spontaneous preterm delivery in women with preterm labor: a retrospective study. BMC Pregnancy Childbirth 2018;18:146.

8. Bhandari A, Bhandari V. Biomarkers in bronchopulmonary dysplasia. Paediatr Respir Rev 2013;14:173-9.

9. Maheshwari A, Schelonka RL, Dimmitt RA, et al. Cytokines associated with necrotizing enterocolitis in extremely-low-birth-weight infants. Pediatr Res 2014;76:100-8.

10. Stocker M, van Herk W, El Helou S, et al. Procalcitoninguided decision making for duration of antibiotic therapy in neonates with suspected early-onset sepsis: a multicentre, randomised controlled trial (NeoPIns). Lancet 2017;390:871-81.

11. Weiss SL, Fitzgerald JC, Balamuth F, et al. Delayed antimicrobial therapy increases mortality and organ dysfunction duration in pediatric sepsis. Crit Care Med 2014;42:2409-17.

12. Tanimura K, Yamada H. Potential Biomarkers for Predicting Congenital Cytomegalovirus Infection. Int J Mol Sci 2018;19:3760.

13. Subspecialty Group of Neonatology, the Society of Pediatric, Chinese Medical Association; Professional Committee of Infectious Diseases, Neonatology Society, Chinese Medical Doctor Association. Zhonghua Er Ke Za Zhi 2019;57:252-7.

14. Burchfield DJ. RE: Management of Neonates Born at $\leq 34$ 6/7 Weeks' Gestation With Suspected or Proven EarlyOnset Bacterial Sepsis. Pediatrics 2019;143:e20190533A.

15. Simonsen KA, Anderson-Berry AL, Delair SF, et al. Earlyonset neonatal sepsis. Clin Microbiol Rev 2014;27:21-47.

16. Sack GH Jr. Serum Amyloid A (SAA) Proteins. Subcell Biochem. 2020;94:421-36.

17. Chauhan N, Tiwari S, Jain U. Potential biomarkers for effective screening of neonatal sepsis infections: An overview. Microb Pathog 2017;107:234-42.

18. van Rossum AM, Wulkan RW, Oudesluys-Murphy AM. Procalcitonin as an early marker of infection in neonates and children. Lancet Infect Dis 2004;4:620-30.

19. Liu C, Fang C, Xie L. Diagnostic utility of procalcitonin as a biomarker for late-onset neonatal sepsis. Transl Pediatr 2020;9:237-42.

20. Zhao FX, Liu GH, Zhang J. Value of IL-6 and IL-8 in the diagnosis of neonatal sepsis. Zhongguo Dang Dai Er Ke Za Zhi 2015;17:1311-5. 
21. Boskabadi H, Zakerihamidi M. Evaluate the diagnosis of neonatal sepsis by measuring interleukins: A systematic review. Pediatr Neonatol 2018;59:329-38.

22. Torii Y, Yoshida S, Yanase Y, et al. Serological screening

Cite this article as: Liu C, Fang C, He Q, Xie L. The value of interleukin-6 (IL-6) within 6 hours after birth in the prompt diagnosis of early-onset neonatal sepsis. Transl Pediatr 2020;9(5):629-635. doi: 10.21037/tp-20-239 of immunoglobulin $M$ and immunoglobulin $\mathrm{G}$ during pregnancy for predicting congenital cytomegalovirus infection. BMC Pregnancy Childbirth 2019;19:205. 